\title{
A survey of the use of complementary medicine by a self-selected community group of Australian women with polycystic ovary syndrome
}

\author{
Susan Arentz ${ }^{1 *}$, Caroline Anne Smith ${ }^{1}$, Jason Anthony Abbott ${ }^{2}$ and Alan Bensoussan ${ }^{1}$
}

\begin{abstract}
Background: Polycystic ovary syndrome (PCOS) is a complex reproductive endocrinopathy affecting up to 20\% of reproductive aged women. Whilst there are effective pharmaceutical treatment options, women with PCOS have expressed a strong desire for alternatives. This study investigates the use and attitudes of women with PCOS towards complementary medicine (CM).

Methods: Women as members of PCOS support groups responded to an anonymous on-line survey which examined rates and patterns of use for CM's, areas of health for use, perceptions of effectiveness, safety and demographic features. Data collection targeted women with PCOS using two consumer support groups. The first group self-selected following direct email to members of a land based consumer support group, the Polycystic Ovary Syndrome Association of Australia (POSAA). The second sample was generated through the electronic social network Facebook, using a snowball technique. Two surveys, identical in content, were collected by cloud based Survey Monkey. Data were described and associations between the variables, 'reasons for use' and 'perceptions of effectiveness' were explored. Non-response bias was assessed using a continuum of resistance model.
\end{abstract}

Results: 493 women participated in the study; 91.1\% response rate from the POSAA group. Over 70\% reported use of complementary medicine, usually nutritional and herbal supplements and $76.6 \%$ of CM users reported consultation with a complementary practitioner. Many participants were using CM to treat PCOS however most were using it to concurrently treat a range of health conditions, describing women's desire for more than single symptom management. Disadvantages for CM use were cited by $71 \%$ of respondents. Women using complementary medicine with specific treatment goals in mind reported greater self-perceived effectiveness, suggesting that informed use may improve women's satisfaction with CM. Adverse reactions were reported by $12.2 \%$ of women and the need for further research into adverse reactions for CM's was identified. Demographic and PCOS characteristics were similar to clinical populations of PCOS and non-response bias was shown as not significant.

Conclusion: This study describes the prevalence of use for complementary medicine by women with PCOS as over $70 \%$ and adds to our understanding of women's experiences with CM and their motivations for use of CM.

\section{Background}

Polycystic ovary syndrome (PCOS) is a complex endocrine disorder affecting up to one in five reproductive aged women [1]. PCOS is a life-long condition associated with significant endocrine, reproductive and psychological morbidity and serious long term health risks [2-4]. The exact cause is unknown however it is believed

\footnotetext{
*Correspondence: s.arentz@uws.edu.au

'National Institute of Complementary Medicine (NICM), University of Western Sydney, Sydney, Australia

Full list of author information is available at the end of the article
}

to result from multiple interactions between genes, the in utero environment and lifestyle behaviors [4]. Current treatments are only moderately effective at controlling symptoms and preventing complications [2] and women with PCOS have expressed strong dissatisfaction towards pharmaceutical therapies. In a survey of women with PCOS $(n=657), 99 \%$ indicated they would prefer to use effective and safe alternative treatments to birth control pills and fertility drugs for the management of their symptoms [5]. 
Women's preferences for complementary treatments coincide with the increasing prevalence for complementary medicine (CM) use. In a critical review of 14 studies, the prevalence of CM use by pregnant women was shown to range up to $87 \%$ [6]. Other studies have demonstrated similar rates of CM use by women attending family planning clinics [7] and by menopausal women [8]. The landmark Australian Women's Health longitudinal study of 11,454 women highlighted that nearly one third of middle aged women had consulted a complementary practitioner (chiropractor, naturopath, acupuncturist or herbalist) within the previous twelve months [9].

To date there have been no investigations into the use of CM by women with PCOS. This study aimed to examine the prevalence of use, the views and patterns of CM use including women's attitudes, views of CM effectiveness and self-reported adverse events from CM.

\section{Method}

Study participants completed an on-line, web-based questionnaire which examined demographic features, patterns of complementary medicine (CM) use, use of CM relating to symptoms of PCOS, perceptions of effectiveness and safety, sources of information on CM and concurrent use of $\mathrm{CM}$ with western medicine.

Women with PCOS were recruited through two approaches; (i) convenience sampling of women belonging to the support/advocacy group Polycystic Ovary Syndrome Association of Australia (POSAA) and (ii) a snowballing technique and response to an invitation posted on the social network site Facebook with a link to the 'University Research for PCOS' Facebook group. Self-referrals were received from the following Facebook groups based in two metropolitan areas in Sydney and New South Wales; PCOS Cysters (121 members); PCOS TTC (333 members); and PCOS Talk (2011 members). This study was approved by the Human Research Ethics Committee at the University of Western Sydney (EC00314) reference H9341.

\section{The research instrument}

We defined CM from an adaptation of the Cochrane Collaboration operational definition [10]. Specifically, our definition for $\mathrm{CM}$ was 'alternative or natural therapies or medicines including taking dietary supplements (herbal, vitamins, minerals and/or food supplements, for example fish oils or barley greens) and or consulting traditional, alternative and complementary practitioners'. Participants were informed about specific types of CMs at the beginning of relevant questions with an extensive list of options and an open question for 'others' at the end. Two prevalence questions included the option 'prescribed by a doctor' to identify mainstream use rather than complementary medicine use. A six point Likert scale ranging from 'daily' to 'as required' enabled participants to report their frequency of use. Participants were asked their reasons for using complementary medicine and could indicate their response to fourteen symptom options. Responses were not limited to one option. Questions about signs and symptoms of PCOS and current management preceded CM use and demographic characteristics. (Additional file 1. The survey instrument.) The research tool was tested on a pilot sample of eight women including four with PCOS.

\section{Survey administration}

The questionnaire was administered using Survey Monkey [11]. An email containing a letter of invitation and an electronic link to the questionnaire was sent to all members of POSAA in November 2011. Two reminders were sent out at six-week intervals. The second approach used to distribute the questionnaire to the community utilized a Facebook group created by the researcher (SA) in February 2012. Facebook groups are virtual communities of choice, linking people with shared interests, attributes or causes. Wall postings occurred bi-monthly during the study administration.

\section{Data analyses}

Analysis was undertaken using the statistical package, SPSS version 21.0 [12]. Categorical responses were reported as numbers and percentages with 95\% confidence intervals. Relationships between variables were explored using Pearson's correlation (two tail) for normally distributed data (use of CM) and Spearman's Rho correlations or Gamma co-efficient for non-parametric measurements (type of CM practitioner). Logistic regression was used to predict whether or not perceptions of effectiveness (dependent variables) were associated with reasons for use (covariates). A value of 0.05 was considered statistically significant with $95 \%$ confidence intervals.

Non-response bias was assessed using the continuum of resistance model; this involves late responders viewed by proxy as non-responders due to similar characteristics [13]. This method of assessment was chosen due to the higher likelihood for early response by women who found the topic salient or interesting [14]. Responses to the survey four months after the final reminder were classified as late responders and compared for difference with early responses to assess the impact of non-response bias.

\section{Results}

\section{Response rate}

Four hundred and ninety three (493) women with PCOS responded to the survey. A response was received from 235 invited members of the consumer support group POSAA (response rate of 91.1\%). An additional 311 women who responded to the survey used the University Research Facebook group. 
The majority of participants were aged between 25 and 34 years, born in Australia, attained tertiary qualifications, were employed and held private health insurance (Table 1).

Women reported multiple symptoms of PCOS. The most common included late menses $(69 \%, \pm 4.31,95 \% \mathrm{CI})$; hirsutism $(66 \%, \pm 4.20,95 \% \mathrm{CI})$; acne $(51 \%, \pm 4.52,95 \% \mathrm{CI})$; insulin imbalances ( $56 \%, \pm 4.63,95 \% \mathrm{CI})$, being overweight $(78 \%$, $\pm 4.46,95 \% \mathrm{CI})$ or very overweight, $(51 \%, \pm 4.61,95 \% \mathrm{CI})$. Other health complaints included depression (45\%, \pm 4.64 , $95 \% \mathrm{CI})$ and infertility $(53 \%, \pm 4.49,95 \% \mathrm{CI})$.

The respondents used various medical therapies to manage their symptoms. Over $65 \%( \pm 4.21,95 \% \mathrm{CI})$ had used the oral contraceptive pill; $62.7 \%( \pm 4.26,95 \% \mathrm{CI})$ had used other pharmaceuticals including ovulation induction, hypoglycaemic and anti-androgen drugs. Medications used to stimulate the ovaries for IVF were reported by $11.8 \%( \pm 2.87,95 \% \mathrm{CI})$.

\section{Use of complementary medicines and therapies}

Three hundred and four, out of 432 women $(70.4 \%$ $\pm 4.3095 \% \mathrm{CI})$ reported using complementary medicine (CM) in the previous 12 months.

Women were asked about their intake habits (Table 2). The most commonly used CM products taken on a daily basis were dietary supplements including vitamins, minerals and food nutrients such as fish oil capsules, and herbal supplements. Two hundred and seven of 432 respondents $(47.9 \% \pm 4.7195 \% \mathrm{CI})$ reported use of more than one type of CM product.

\section{Complementary practitioner visits}

Consultations with acupuncturists, chiropractors, naturopaths, and massage therapists in the previous twelve months were reported by 233 of $304 \mathrm{CM}$ users $(76.6 \% \pm$ 4.76 95\% CI) (Table 3) . A smaller number of women also consulted medical doctors for acupuncture treatment or integrative medicine which was provided within the general medical setting. Nearly half of respondents using $\mathrm{CM}$ consulted multiple CM practitioners during the previous twelve months, usually an acupuncturist and naturopath $(\mathrm{n}=47,15.5 \% \pm 4.0795 \% \mathrm{CI})$ or an acupuncturist and chiropractor $(n=41,13.5 \% \pm 3.8495 \%$ CI).

\section{Reasons for using Complementary Medicine (CM)}

Many women were using CM to treat PCOS $(n=199$, $65.5 \%, \pm 5.34,95 \% \mathrm{CI}$ ) however the majority of participants reported using $\mathrm{CM}$ to assist with improving more than one aspect of their health $(n=277,76.7 \%, \pm 4.75$, 95\% CI). The most common reasons for use were to improve general wellbeing $(n=135,44.4 \% \pm 5.5995 \% \mathrm{CI})$; and to treat PCOS symptoms and infertility $(n=102$, $33.6 \%, \pm 5.31,95 \% \mathrm{CI})$, and treat PCOS and reduce depression $(\mathrm{n}=92,30.3 \% \pm 5.1795 \% \mathrm{CI})$.

Table 1 Demographic characteristics of respondents

\begin{tabular}{lrrr}
\hline & $\mathbf{N}=\mathbf{4 9 3}$ & $\mathbf{\%}$ & $\mathbf{9 5 \%} \mathbf{C l}$ \\
\hline Age & & & \\
17 or less & 3 & 0.7 & \pm 0.79 \\
$18-24$ & 72 & 17.0 & \pm 3.58 \\
$25-29$ & 126 & 29.7 & \pm 4.35 \\
$30-34$ & 111 & 26.2 & \pm 4.19 \\
$35-40$ & 71 & 16.7 & \pm 3.55 \\
$41-44$ & 25 & 5.9 & \pm 2.24 \\
$45+$ & 16 & 3.8 & \pm 1.82 \\
Missing data & 69 & &
\end{tabular}

Education

Completed high School

Yes

Missing data

371

69

Completed tertiary education

Yes

Missing data

Qualification from TAFE

Private institution Diploma

University degree

Missing data

Ethnicity- country of birth

Australia

Other

Missing data

Employment

Home duties

Self-employed

Student

Employed part time

Employed full-time

Other

Missing data

Private Health Insurance

Yes

Missing data

Missing data for age, complete high school, tertiary education, ethnicity, employment and health insurance was $14.0 \%$. Missing data for qualifications $20.1 \%$.

\section{Views about CM}

Twenty seven percent of CM users $(n=82, \pm 5.99,95 \%$ CI) indicated they thought $\mathrm{CM}$ used alone was effective management for PCOS and 24.7\% ( $\mathrm{n}=75, \pm 4.85,95 \% \mathrm{CI})$ reported that CM was effective for PCOS in conjunction with diet and exercise. Participant's indicated they thought $\mathrm{CM}$ was effective for treating more than one condition, 
Table 2 Types of ingestible complementary medicine taken by participants and frequency of use

\begin{tabular}{|c|c|c|c|c|c|c|c|}
\hline \multirow[t]{2}{*}{ Complementary medicine use $N=432$} & \multicolumn{2}{|c|}{ Daily use } & \multicolumn{2}{|c|}{ Weekly use } & \multicolumn{2}{|c|}{ Total use } & \multirow[t]{2}{*}{$95 \% \mathrm{Cl}$} \\
\hline & N & $\%$ & $\mathrm{~N}$ & $\%$ & $\mathrm{~N}$ & $\%$ & \\
\hline Nutritional supplements, including vitamins, minerals, nutrients from food such as fish oil. & 192 & 44.4 & 92 & 21.3 & 246 & 56.9 & \pm 4.67 \\
\hline Herbal medicines including herbal tea (for therapeutic reasons), tablets and liquids. & 138 & 31.94 & 55 & 12.73 & 181 & 41.9 & \pm 4.65 \\
\hline Vitamins and/or minerals prescribed by a medical doctor & 62 & 14.4 & 10 & 2.3 & 63 & 14.6 & \pm 3.33 \\
\hline Multiple forms of CAM products & 137 & 31.71 & 70 & 16.2 & 207 & 47.9 & \pm 4.71 \\
\hline Total use & 238 & 55.1 & 179 & 41.4 & 304 & 70.4 & \pm 4.3 \\
\hline
\end{tabular}

- Categories were not mutually exclusive, women may have been using more than one product/modality with variable patterns of use.

- Cl confidence interval.

( $\mathrm{n}=115,37.8 \%, \pm 5.44,95 \% \mathrm{CI})$ including improved general well-being $(\mathrm{n}=96,31.6 \%, \pm 5.2395 \% \mathrm{CI})$, sleep ( $\mathrm{n}=51,16.8 \%, \pm 4.2295 \% \mathrm{CI})$ and weight loss $(\mathrm{n}=48$, $15.8 \%, \pm 4.0995 \% \mathrm{CI})$. A smaller number of women considered CM was effective for infertility $(n=33$, $10.9 \% \pm 3.595 \% \mathrm{CI})$.

Significant relationships were found between using $\mathrm{CM}$ to treat specific conditions and perceptions of effectiveness. Use of CM to treat PCOS ( $n=199)$, was associated with effectiveness of CM by 40.8\% (PCC 4.64, p = 0.001) of participants. Use of $\mathrm{CM}$ as treatment for infertility ( $\mathrm{n}=102$ ) was associated with effectiveness by $32.4 \%$ (PCC 3.84, $\mathrm{p}=0.001$ ) of women and the treatment goal, 'to improve wellbeing' $(\mathrm{n}=134)$, was associated with $71.6 \%$ (PCC 4.04, $\mathrm{p}=0.001$ ) of women reporting effectiveness.

Predictions were found for using CM to treat PCOS and perceptions of effectiveness for PCOS (odds ratio (OR) 24.8, CI 11.1-55.3, $\mathrm{p}=0.0001$ ); infertility, (OR 18.8, CI 7.9-44.8, $\mathrm{p}=0.0001)$ and for improved wellbeing (OR 8.8, CI 5.2-14.9, $\mathrm{p}=0.0001$ ).

\begin{tabular}{|c|c|c|c|}
\hline Use of $C M$ & $\mathrm{~N}$ & $\%$ & $95 \% \mathrm{Cl}$ \\
\hline \multicolumn{4}{|l|}{$N=304$} \\
\hline Consultations with CM practitioners & 233 & 76.6 & \pm 4.76 \\
\hline More than one type of CM practitioner & 136 & 44.7 & \pm 5.59 \\
\hline Naturopath & 118 & 38.8 & \pm 5.48 \\
\hline Chiropractor & 103 & 33.9 & \pm 5.32 \\
\hline Acupuncturist & 88 & 28.9 & \pm 5.1 \\
\hline Massage therapist & 79 & 25.1 & \pm 4.87 \\
\hline Osteopath & 41 & 13.5 & \pm 3.84 \\
\hline Reflexologist, aromatherapist, iridologist & 36 & 11.8 & \pm 3.63 \\
\hline Homoeopath & 34 & 11.2 & \pm 3.55 \\
\hline Medical doctor for integrative medicine & 27 & 8.9 & \pm 3.2 \\
\hline Kinesiologist & 20 & 6.6 & \pm 2.79 \\
\hline Medical doctor for acupuncture & 14 & 4.6 & \pm 2.35 \\
\hline
\end{tabular}

- Footnote: 301 women responded to this question, missing data $=3$. - Responses were not limited to single options with 136 providing more than one response for type of $\mathrm{CM}$ practitioner.

- $\mathrm{Cl}$, confidence interval.
Advantages for CM were reported by $52.5 \%(\mathrm{n}=259, \pm$ $4.41,95 \% \mathrm{CI})$. These included perceived naturalness of CM ( $\mathrm{n}=157,60.6 \% \pm 5.95,95 \% \mathrm{CI})$, the complementary nature of treatment $(n=120,46.3 \% \pm 6.07,95 \% C I)$, perceived low risk of side effects $(n=104,40.2 \%, \pm 5.97,95 \%$ $\mathrm{CI})$, increased self- responsibility $(\mathrm{n}=81,31.3 \%, \pm 5.65$, $95 \% \mathrm{CI})$ and the holistic nature of CM ( $\mathrm{n}=75,29 \%, \pm 5.52$ 95\% CI).

Disadvantages associated with CM use were cited by 354 women $(71.8 \%, \pm 3.9795 \% \mathrm{CI})$. These included CM being too expensive $(n=187,52.8 \% \pm 5.2,95 \% \mathrm{CI})$, lack of research into CM $(\mathrm{n}=175,49.4 \% \pm 5.2195 \% \mathrm{CI})$ and 163 women $(46.0 \% \pm 5.19,95 \% \mathrm{CI})$ were not confident about a benefit from CM. A small number of women (13.6\%, $\pm 3.57,95 \% \mathrm{CI})$ indicated they were not confident to use $\mathrm{CM}$ in conjunction with other pharmaceuticals.

\section{Adverse reactions and $\mathrm{CM}$}

Participants were asked about any adverse reactions or negative experiences with their use of CM. Adverse reactions to nutritional and herbal supplements, Traditional Chinese Herbal Medicine (TCHM) and acupuncture were reported by thirty seven CM users $(12.2 \%, \pm 3.68,95 \% \mathrm{CI})$. Nine reactions were to vitamins, seven to fish oil supplements, six to TCM including two to acupuncture, two to herbal teas and five to vitamin supplements prescribed by a medical practitioner. Eleven adverse reactions were reported including longer menstrual cycles, changes to bowel habits, sleep disturbances and headaches.

\section{Assessment for non-response bias and selection bias}

When testing for non-response bias for early and late responders no significant differences were noted in the demographic characteristics, symptoms of PCOS and prevalence for use of CM between any of the surveyed groups.

\section{Discussion}

The use of complementary medicine by women has increased in recent years. We present the first survey of women with PCOS and report high use of CM, with nearly three out of four women reporting use and nearly half reporting use of multiple CM's. This study demonstrates 
that women seek more than the management of symptoms associated with PCOS when using CM's and that wellbeing, reproductive and mental health are important aspects of care. Our findings are limited by the sampling framework and the referral of participants through support groups, since women with PCOS whom have not sought support through POSAA or electronic social media, or women with PCOS in the community who have not been diagnosed [1] were not represented in this study.

We found women have similar preferences for the types of CM use to those reported in reproductive health settings where nutritional and herbal supplements were the most commonly reported CM (comparatively, 56\% for women attending family planning clinics and $78 \%$ of women attending fertility clinics) $[7,15]$. However, our findings differ to studies showing recently increased preferences for acupuncture compared to naturopaths $[7,16]$. These differences may be explained by our study focusing only on women with PCOS, the definition of $\mathrm{CM}$ modalities used or influenced by recent statutory registration of acupuncture practitioners in Australia.

Most women perceived the effectiveness of CM as at least somewhat helpful. This aligns with findings from other surveys of women attending family planning clinics [7], pregnant women [17] and middle aged women [8]. Our data show women's perceptions of effectiveness were increased when they used CM with specific treatment goals in mind. Little is known about how women with PCOS acquire information and make decisions regarding suitable CM's. This extended beyond the scope of our study however our findings demonstrate the value of informed CM treatment decisions and highlight the decision process for $\mathrm{CM}$ as an area of future research need. The main disadvantage for CM cited in this study was the lack of research which reiterates women's need for unbiased information to support their CM decisions.

Our data corresponds with findings reporting women have reduced recognition for potential hazards for $\mathrm{CM}$ $[7,18,19]$. Some herbal medicines taken in conjunction with pharmaceuticals may have adverse effects. Hypericum perforatum (St Johns wort) for example, has been shown to reduce pharmaceutical effectiveness [20-22]. Importantly, our findings report participant's high use for herbal medicines and high use of pharmaceuticals including the oral contraceptive pill (over 65\%). Future research is needed to examine concurrent use of pharmaceuticals and $\mathrm{CM}$ by women with PCOS, and the rate of adverse events attributable to reduced pharmaceutical effectiveness.

The rate of adverse events reported here was not as low as in other studies. A survey of TCM (Chinese herbal medicine and acupuncture) practitioners demonstrated adverse reaction rates of one per 633 consultations [23]. Comparatively there were six adverse reactions for TCM out of 304 CM users. Our data include self-reported adverse reactions by selfprescribing consumers with potentially poorly informed treatment choices. This may explain the higher rates of adverse reactions in this case. On the other hand, adverse reactions reported by TCM practitioners may be at risk of reporting bias. Our research highlights that women with PCOS do experience adverse reactions when using $\mathrm{CM}$, despite perceptions of low risk.

This study does have some methodological limitations. We were unable to directly assess non-response and selection bias for the Facebook group. The referral to the survey by administrators of other Facebook support groups limited our ability to gauge unsolicited invitations and data were not available for assessment using the continuum of resistance model. Generalizations are limited to computer literate women with English language skills and other groups of women may be underrepresented. However the results may be generalisable to women with PCOS. Women reported similar prevalence for key PCOS signs and symptoms to clinical populations for hirsutism (55.6\% [1], 66\% [24] and 75\% [25]) and menstrual irregularities $(62.5 \%$ [1], 66\% [24] and $89 \%$ [25]). This suggests that the respondents resemble a clinical population with PCOS. We also attained a good response rate without response bias from women with PCOS in a non-clinical setting, not restricted by geographical location. This survey had high completion rates and suggests that the methods were acceptable to women. For these reasons our data are likely to reflect the authentic views of women with PCOS in the community. This research was focused on the use of CM by women with PCOS and differentiates CM from other lifestyle practices; however women often use CM within the context of other lifestyle behaviors, including diet and exercise. The prevalence, patterns and attitudes towards diet and exercise of women with PCOS was beyond the scope of the present article.

\section{Conclusion}

Complementary medicine may present a treatment option with high acceptance by women; this study is an important step in developing our understanding of this group of women's views and patterns of CM use.

\section{Additional file}

Additional file 1: The survey instrument.

Competing interests

The authors declare that they have no competing interests. SA is a doctoral research student and CAS, JAB and $A B$ are supervisory personnel. The submission processing fee was provided by the University of Western Sydney as part of an academic institutional membership. 


\section{Authors' contributions}

$S A, C S, J A$ and $A B$ conceived of the study and participated in its design and coordination. SA administered the study including development of sampling strategy, recruitment, data collection, and data analyses. SA and CS participated in liaison with community groups. JA and AB reviewed the quality of data and data analyses. SA, CS, JA and AB designed and edited the tables. All authors read, edited and approved the final manuscript.

\section{Acknowledgements}

The authors would like to acknowledge the contribution of the participants in this study and the statistical guidance provided by statistician, Dr Paul Fahey of the University of Western Sydney. We would also like to acknowledge the valuable support of president of POSAA, Veryan McAlistair, the volunteers and the assistance of Facebook PCOS support group administrators Jamie Russell and Kristina Judd.

\section{Author details}

'National Institute of Complementary Medicine (NICM), University of Western Sydney, Sydney, Australia. ${ }^{2}$ School of Women's and Children's Health, University of New South Wales, Randwick, Australia.

Received: 8 August 2014 Accepted: 27 November 2014

Published: 6 December 2014

\section{References}

1. March WA, Moore VM, Willson KJ, Phillips DIW, Norman RJ, Davies MJ: The prevalence of polycystic ovary syndrome in a community sample assessed under contrasting diagnostic criteria. Hum Reprod 2010, 25(2):544-551.

2. ESHRE: Consensus on women's health aspects of polycystic ovary syndrome (PCOS). Hum Reprod 2012, 27(1):14-24.

3. Teede H, Deeks A, Moran L: Polycystic ovary syndrome: a complex condition with psychological, reproductive and metabolic manifestations that impacts on health across the lifespan. BMC Med 2010, 8(1):41.

4. Norman RJ, Dewailly D, Legro RS, Hickey TE: Polycystic ovary syndrome. Lancet 2007, 9588:685-697.

5. Sills ES, Perloe M, Tucker MJ, Kaplan CR, Genton MG, Schattman GL: Diagnostic and treatment characteristics of polycystic ovary syndrome: descriptive measurements of patient perception and awareness from 657 confidential self-reports. BMC Womens Health 2001, 1(1):3.

6. Adams J, Lui CW, Sibbritt D, Broom A, Wardle J, Homer C, Beck S: Women's use of complementary and alternative medicine during pregnancy: a critical review of the literature. Birth 2009, 36(3):237-245.

7. Smith CA, Bateson DJ, Weisberg E: A survey describing the use of complementary therapies and medicines by women attending a family planning clinic. BMC Complement Altern Med 2013, 13(1):224.

8. Gollschewski S, Anderson D, Skerman H, Lyons-Wall P: Associations between the use of complementary and alternative medications and demographic, health and lifestyle factors in mid-life Australian women. Climacteric 2005, 8(3):271-278.

9. Sibbritt DW, Adams J, Young AF: A longitudinal analysis of mid-age women's use of complementary and alternative medicine (CAM) in Australia, 1996-1998. Women Health 2005, 40(4):41-56.

10. Wieland LS, Manheimer E, Berman BM: Development and classification of an operational definition of complementary and alternative medicine for the Cochrane collaboration. Altern Ther Health Med 2011, 17(2):50.

11. SurveyMonkey web based survey software, Finley R: SurveyMonkey web based survey software. 2013. [cited 3012-3; Internet survey software using Listserv ]. Available from: www.surveymonkey.com.

12. IBM: SPSS version 21, Statistics for Windows. IBM Corporation. Armonk NY; 2013.

13. Boshuizen HC, Viet AL, Picavet HSJ, Botterweck A, Van Loon AJM: Non-response in a survey of cardiovascular risk factors in the Dutch population: determinants and resulting biases. Public Health 2006, 120(4):297-308

14. Porter SR, Whitcomb ME: Non-response in student surveys: the role of demographics, engagement and personality. Res High Educ 2005, 46(2):127-152.

15. Stankiewicz M, Smith C, Alvino H, Norman R: The use of complementary medicine and therapies by patients attending a reproductive medicine unit in South Australia: a prospective survey. Aust New Zeal J Obstet Gynaecol 2007, 47(2):145-149.
16. Frawley J, Adams J, Sibbritt D, Steel A, Broom A, Gallois C: Prevalence and determinants of complementary and alternative medicine use during pregnancy: Results from a nationally representative sample of Australian pregnant women. Aust New Zeal J Obstet Gynaecol 2013, 53(4):347-352.

17. Skouteris $\mathrm{H}$, Wertheim $\mathrm{E}$, Rallis $\mathrm{S}$, Paxton $\mathrm{S}$ : Use of complementary and alternative medicines by a sample of Australian women during pregnancy. Aust N Z J Obstet 2008, 48:384-390.

18. Tiran D: The use of herbs by pregnant and childbearing women: a risk-benefit assessment. Compl Ther Nurs Midwifery 2003, 9(4):176-181.

19. Nordeng H, Havnen GC: Use of herbal drugs in pregnancy: a survey among 400 Norwegian women. Pharmacoepidemiol Drug Saf 2004, 13(6):371-380.

20. Pfrunder A, Schiesser M, Gerber S, Haschke M, Bitzer J, Drewe J: Interaction of St John's wort with low-dose oral contraceptive therapy: a randomized controlled trial. Br J Clin Pharmacol 2003, 56(6):683-690.

21. Hennessy M, Kelleher D, Spiers JP, Barry M, Kavanagh P, Back D, Mulcahy F, Feely J: St John's Wort increases expression of P-glycoprotein: Implications for drug interactions. Br $」$ Clin Pharmacol 2002, 53(1):75-82.

22. Mills S, Bone K: The essential guide to herbal safety. St Louis Missouri USA: Elsevier Health Sciences; 2005.

23. Bensoussan A, Myers SP, Carlton A-L: Risks associated with the practice of traditional Chinese medicine: an Australian study. Arch Fam Med 2000, 9(10):1071.

24. Balen AH, Balen AH, Conway GS, Kaltsas G, Techatraisak K, Manning PJ, West C, Jacobs HS: Andrology: Polycystic ovary syndrome: the spectrum of the disorder in 1741 patients. Hum Reprod 1995, 10(8):2107-2111.

25. Ching H, Burke V, Stuckey B: Quality of life and psychological morbidity in women with polycystic ovary syndrome: body mass index, age and the provision of patient information are significant modifiers. Clin Endocrinol (Oxf) 2007, 66(3):373-379.

doi:10.1186/1472-6882-14-472

Cite this article as: Arentz et al:: A survey of the use of complementary medicine by a self-selected community group of Australian women with polycystic ovary syndrome. BMC Complementary and Alternative Medicine 2014 14:472.

\section{Submit your next manuscript to BioMed Central and take full advantage of:}

- Convenient online submission

- Thorough peer review

- No space constraints or color figure charges

- Immediate publication on acceptance

- Inclusion in PubMed, CAS, Scopus and Google Scholar

- Research which is freely available for redistribution 